\title{
Advances in mass spectrometry imaging enabling observation of localised lipid biochemistry within tissues
}

Citation for published version (APA):

Bowman, A. P., Heeren, R. M. A., \& Ellis, S. R. (2019). Advances in mass spectrometry imaging enabling observation of localised lipid biochemistry within tissues. Trac-Trends in Analytical Chemistry, 120, [115197]. https://doi.org/10.1016/j.trac.2018.07.012

Document status and date:

Published: 01/11/2019

DOI:

10.1016/j.trac.2018.07.012

Document Version:

Publisher's PDF, also known as Version of record

Document license:

Taverne

Please check the document version of this publication:

- A submitted manuscript is the version of the article upon submission and before peer-review. There can be important differences between the submitted version and the official published version of record.

People interested in the research are advised to contact the author for the final version of the publication, or visit the DOI to the publisher's website.

- The final author version and the galley proof are versions of the publication after peer review.

- The final published version features the final layout of the paper including the volume, issue and page numbers.

Link to publication

\footnotetext{
General rights rights.

- You may freely distribute the URL identifying the publication in the public portal. please follow below link for the End User Agreement:

www.umlib.nl/taverne-license

Take down policy

If you believe that this document breaches copyright please contact us at:

repository@maastrichtuniversity.nl

providing details and we will investigate your claim.
}

Copyright and moral rights for the publications made accessible in the public portal are retained by the authors and/or other copyright owners and it is a condition of accessing publications that users recognise and abide by the legal requirements associated with these

- Users may download and print one copy of any publication from the public portal for the purpose of private study or research.

- You may not further distribute the material or use it for any profit-making activity or commercial gain

If the publication is distributed under the terms of Article $25 \mathrm{fa}$ of the Dutch Copyright Act, indicated by the "Taverne" license above, 


\title{
Advances in mass spectrometry imaging enabling observation of localised lipid biochemistry within tissues
}

\author{
Andrew P. Bowman, Ron M.A. Heeren, Shane R. Ellis* \\ The Maastricht Multimodal Molecular Imaging Institute (M4I), Division of Imaging Mass Spectrometry, Maastricht University, 6229 ER Maastricht, The \\ Netherlands
}

\section{A R T I C L E I N F O}

\section{Article history:}

Available online 30 July 2018

\section{Keywords:}

Lipids

Lipidomics

Mass spectrometry imaging

Biochemistry

Enzymes

Isotope labelling

MALDI

SIMS

DESI

\begin{abstract}
A B S T R A C T
There are numerous reports demonstrating the heterogeneous spatial distributions of lipids throughout biology using mass spectrometry imaging (MSI). However, despite these and the biological significance of lipid alterations there remains a surprising lack of understanding regarding the underlying origins of the spatial distributions detected with MSI. With the rapid improvements in analytical capabilities of MSI instrumentation, it is now at the point that these biological questions can begin to be addressed. In this review we focus on (i) work providing insight into the biochemical origins of lipid spatial distributions, with an emphasis on the role of localised enzymatic processes in determining lipid compositions; (ii) use of stable isotope labelling to spatially-resolve the kinetics of lipid synthesis; and (iii) current challenges faced by the lipid MSI community and new analytical methods to overcome them. Combined, these topics highlight the added biological information that can be acquired from the MSI of lipids.
\end{abstract}

() 2018 Elsevier B.V. All rights reserved.

\section{Introduction}

The continuous and rapid improvements in mass spectrometry (MS) technology is strongly correlated with the increased appreciation and understanding of the critical and complex roles performed by lipids in biology [1,2]. While the ever-improving resolving power and structural elucidation capabilities of MS-based lipidomics have shed light onto the sheer complexity of the lipidome, both with respect to compositional and structural variations, significant analytical challenges are still faced [3]. To date, the majority of lipidomics studies are performed on extracts following sample homogenization. While these approaches have proven immensely valuable they necessitate the loss of information regarding the spatial context of a lipid in a heterogeneous tissue or cell. As a consequence, information relating to the local microenvironment in which a lipid is present is sacrificed, and significant, but highly localised, lipid alterations may be diluted beyond the detection limit in the extract.

Over the last decade, mass spectrometry imaging (MSI) has emerged as a powerful analytical method to probe the spatial distributions of many lipid classes throughout biological tissues [4,5].

\footnotetext{
* Corresponding author.

E-mail address: s.ellis@maastrichtuniversity.nl (S.R. Ellis).
}

A key advantage of MSI over other imaging modalities is the ability to perform unlabelled imaging of many molecules in parallel, facilitating simultaneous detection of numerous lipid species having distinct mass-to-charge values. From a lipidomics standpoint one can thus think of MSI as performing a $\mathrm{MS}^{1}$-level shotgun lipidomics experiment at each pixel. This can be achieved using a variety of desorption/ionisation methods, with Matrix-Assisted Laser Desorption/Ionisation (MALDI) [5], Secondary Ion Mass Spectrometry (SIMS) [6] and Desorption Electrospray Ionisation (DESI) [7] representing the most popular approaches.

Lipids present a highly promising class of molecules to study by MSI, due to both the high abundance and ionisation efficiency of many lipid species, and their implications in many biological processes. Furthermore, unlike other molecular classes like proteins, lipids have arguably the most straightforward sample preparation procedures, making their detection often reproducible and robust. By exploiting the untargeted nature of MS, it is possible to investigate changing lipid profiles throughout different tissues and cell types. Heterogeneity in lipid profiles is observed in virtually all MSI studies, highlighting the many and specific roles performed by individual lipid species, and the valuable information obtainable from their spatial distributions. This has led to the emergence of MSI as a potentially powerful diagnostic tool, capable of identifying tissuetype changes in lipid composition and making diagnostic predictions based on localised lipid composition [8]. To illustrate, 


\begin{tabular}{|llll|}
\hline Abbreviations & MSI & Mass spectrometry imaging \\
& & MUL & Monounsaturated lipids \\
AA-CL & Arachidonic acid containing lipids & OzID & Ozone-induced dissociation \\
DAG & Diacylglyceride & PA & Phosphatidic acid \\
DESI & Desorption electrospray ionization & PC & Phosphatidylcholine \\
ESI & Electrospray ionization & PE & Phosphatidylethanolamine \\
FTICR & Fourier-transform ion cyclotron resonance & PI & Phosphatidylinositol \\
GalCer & Galactosylceramide & PS & Phosphatidylserine \\
h.p.i & Hours post-infection & SCD1 & Stearoyl-CoA desaturase-1 \\
HBV & Hepatitis B virus & SIMS & Secondary ion mass spectrometry \\
LC & Liquid Chromatography & SM & Sphingomyelin \\
MAG & Monoacylglyceride & SMS2 & Sphingomyelin synthase 2 \\
MALDI & Matrix-assisted laser desorption/ionization & TAG & Triacylglyceride \\
MS/MS & Tandem mass spectrometry & TOF & Time-of-flight \\
MS & Mass spectrometry & & \\
\hline
\end{tabular}

changes in local lipid compositions have been shown to enable differentiation of brain tumor sub-types [9] and the mapping of spatially-dependent chemical alterations in liver disease [10], while lipid profiles also show much promise for real-time annotation of tissue types during surgery [11,12]. This latter approach is typical of many lipid MSI studies that seek to determine the alterations in global profiles throughout different tissue regions without necessarily needing to know the identity or implications of the detected lipids. However, to understand the biological meaning behind MSI data, identification of the lipid species is required.

Despite the ever-increasing utility of lipid MSI as an (bio) analytical technique, it is remarkable that there exists little knowledge as to why lipids exhibit different spatial distributions throughout tissues or with a change of biochemical state (e.g., disease). In many MSI studies, accurate mass and tandem mass spectrometry (MS/MS) are performed to identify some of the observed lipids. However, due in part to a general lack of understanding regarding the roles of individual lipid species in biology, it has been difficult to interpret lipid spatial distributions in terms of biological processes. To move beyond simply visualising altered spatial distributions, it is becoming increasingly important to understand the biochemical origin of lipid MSI results. This would enable researchers to place images into a biological context and understand both the cause and consequence of spatially altered lipid compositions. With advances in sensitivity, spatial resolution, and chemical specificity, MSI technology is now at the point where it can begin to address these paramount questions. In this review, we describe MSI work focusing not only on lipid spatial distributions, but also on beginning to elucidate the underlying processes giving rise to lipid MSI data. We also describe advances in MSI technology that provide new opportunities for MSI including visualising local synthesis kinetics, improved lipidome coverage and sensitivity along with critical analytical challenges that must be addressed to enable imaging of structurally-defined lipid species.

\section{Analytical methods for MSI}

In this section we briefly describe relevant analytical aspects of the key desorption/ionisation techniques used for lipid MSI.

\section{1. $M A L D I$}

MALDI is the most prominent method used for lipid MSI. It offers high sensitivity for many important lipid classes and can be coupled to most mass analysers, enabling both high mass accuracy and structural analysis via MS/MS of detected lipids. The requirement for a matrix requires careful consideration in regards to sample preparation, especially if high spatial resolution is required. Further details of MALDI-MSI sample preparation are provided in Refs. $[13,14]$. The classes of lipids detected can be somewhat tuned via matrix selection; however, in most cases positive-ion mode spectra are dominated by phosphatidylcholine (PC) and sphingomyelin (SM), while negative-ion spectra are dominated by acidic phospholipids and sphingolipids (e.g., sulfatides) [5]. Although termed a "soft" approach, MALDI can still result in some in-source fragmentation which can lead to erroneous assignment of detected ions as endogenous lyso lipids, phosphatidic acid (PA), dehydrated diacylglycerides (DAG) or free fatty acids that may readily form following phospholipid fragmentation. Commercial MALDI MSI instruments now offer throughput approaching 40 pixels/second and spatial resolutions down to $10 \mu \mathrm{m}$, while experimental instruments now approach one micron [15].

\subsection{SIMS}

SIMS offers the highest spatial resolution of any MSI technique. Using traditional atomic ion beams, spatial resolutions as low as several hundred nanometers have been achieved for lipid imaging $[6,16]$. However, the resulting high energy desorption/ionisation process results in severe fragmentation meaning intact lipid species are barely observed, making true lipidome imaging and interpretation difficult. This has been partly addressed with application of soft cluster sources such as $\mathrm{C}_{60}^{+}$that offer softer ionisation conditions and enable detection of intact lipid species [6]. When deployed on instruments enabling continuous beam operation spatial resolutions as low as $300 \mathrm{~nm}$ have been reported [17]. More recently, gas cluster ion beams (e.g., $\mathrm{Ar}_{4000}$ clusters) have further improved the analytical capability of SIMS for lipid MSI [18]. These offer the softest ion generation conditions of any SIMS approach, but are to-date limited to spatial resolutions in the low micron range and can still result in greater fragmentation compared to MALDI [18]. Thus, consideration of these effects is essential to avoid misinterpretation of fragment ions as biologically significant lipids. Nonetheless, the ability to generate intact lipid ions from tissues has led to the development of SIMS identification approaches using both MS/MS and high mass resolution systems [19-21].

\subsection{DESI}

DESI is the most widespread ambient ionisation technique used for lipid MSI [22]. DESI facilitates MSI at atmospheric pressure 
without the need for an external matrix. It is a soft ionisation method (similar to ESI), enabling detection of a broad range of lipid classes with minimal fragmentation. Additionally, it can be coupled to a variety of mass analysers enabling high mass resolution and MS/MS for lipid identification. The typical spatial resolution is $100-250 \mu \mathrm{m}$, which is sufficient to resolve many distinct tissue regions characterised by different lipid compositions [23]. A unique aspect of DESI is the ability to perform in-situ derivatisation during the desorption/ionisation step, which can enable detection of poorly ionised lipid classes such as cholesterol [24].

\section{Applications of MSI to localised lipid biochemistry}

Many lipid MSI studies have focused on elucidating the altered lipid metabolism within cancers. In a MALDI MSI study utilizing Fourier Transform Ion Cyclotron Resonance (FTICR) MSI of lipids detected from six different types of cancer (breast, lung, colorectal, esophageal, gastric, and thyroid), Guo et al. observed elevated signals of monounsaturated lipids (MUL) in cancerous tissues [25]. All six cancer types across more than 100 different patients demonstrated a conserved set of lipid alterations, including PCs $32: 1,34: 1$, and 36:1 showing elevated signals in cancerous tissue relative to their saturated analogues. Interestingly, different cancers were found to overexpress specific MUL relative to others. For example, the $\mathrm{PC}(32: 1): \mathrm{PC}(32: 0)$ ratio was increased in all cancer types except thyroid cancer, whereas the $\mathrm{PC}(36: 1): \mathrm{PC}(36: 0)$ ratio was elevated in all cancers except colorectal cancer (Fig. 1a). Concomitantly, polyunsaturated lipids, notably PC and phosphatidylinositol (PI) 38:4, exhibited a marked decrease in concentration in several of the cancers. The source of these significant lipid alterations was determined by studying the associated expression of enzymes related to lipid synthesis [25]. A significant elevation in expression of fatty acid synthase, stearoyl-CoA desaturase-1 (SCD1), and choline kinase $\alpha$ in the cancers was observed and correlated well with observed MSI data (Fig. 1b), suggesting lipid distributions were the result of localised enzymatic processes [25]. This correlates well with the understanding that SCD1 is the key enzyme of the rate limiting step in the synthesis of monounsaturated fatty acids [26]. Similar SCD1 mediated alterations in monounsaturated PC lipids relative to saturated analogues have also been reported using MSI in breast cancer [27] and, tentatively, in lymphoma [28]. In addition to changes in (un)saturation, negative-ion mode MALDI MSI has shown sulfatides to be elevated in ovarian cancers [29]. Intriguingly, the increased sulfatide levels were not homogenous within the tumor, but instead localised to the tumor epithelium. Sulfatide levels were strongly correlated with a significant increase in both galactolsylceramide (GalCer) synthase (11-fold) and galactose-3-O-sulfotransferase 1 (3.5-fold) relative to normal ovarian stromal tissue, both of which are involved in sulfatide synthesis [29]. This provides a probable insight into the underlying biochemical origin of spatially-resolved sphingolipid metabolism.

MALDI MSI of both liver and kidney tissues sourced from wild type and sphingomyelin synthase 2 (SMS2) deficient mice fed both a normal (ND) and high fat (HFD) diets has shed further light onto alterations in sphingolipid metabolism [8]. SMS2 is a key enzyme involved in the conversion of ceramides into SM. In all mice, potassiated $\mathrm{SM}(\mathrm{d} 18: 1 / 16: 0)$ was localised at the border of the renal cortex and medulla. In contrast, $[\mathrm{SM}(\mathrm{d} 18: 1 / 22: 0)+\mathrm{K}]^{+}$was localised in the medulla, with lower levels observed in the SMS2knockout mice [8]. Moreover, $[\mathrm{SM}(\mathrm{d} 18: 1 / 24: 0)+\mathrm{K}]^{+}$and $[\mathrm{SM}(\mathrm{d} 18: 1 / 24: 1)+\mathrm{K}]^{+}$were localised in the renal cortex and exhibited reduced levels upon high fat diet and SMS2 deficiency, thus pointing to a role of SMS2 in region-specific SM synthesis in the kidney.
Enzymatic processes can also lead to the production of bioactive lipids that propagate the effects of disease/infection. In this context, several MSI studies have focused on the local synthesis and conversion of arachidonic acid containing lipids (AA-CLs). Scott et al [30] showed the breakdown of AA-CLs upon Francisella novicida infections in mice using MALDI TOF MSI and MALDI FTICR MSI. The infection was spatially visualised throughout mouse spleens via the infection-specific Lipid A molecule, which was distributed throughout the red pulp and detected from $36 \mathrm{~h}$ post-infection (h.p.i.) (Fig. 2a). Spatial alterations in the host lipidome were also detected, and revealed a high initial abundance of $\operatorname{PI}(16: 0 / 20: 4)$ in the white pulp. At 48 h.p.i., a drastic decrease in $\mathrm{PI}(16: 0 / 20: 4)$ was observed and associated with the onset of sepsis and breakdown of the white pulp followed by necrosis at 60 h.p.i (Fig. 2b) [30]. Similar reductions in other AA-CLs were also observed and confirmed with quantitative LC-MS/MS (Fig. 2c-f). The decrease in AA-CLs was shown to be the outcome of AA detachment from phospholipids via an increased activity of cytosolic phospolipase-A2 in the infection (Fig. 2g). Critically, this release of AA was associated with significant elevation in cycloxegenase-2 (Fig. 2h), leading to the formation of the highly inflammatory eicosanoid prostaglandin E2 (quantified by LC-MS/MS). This dramatic and rapid generation of inflammatory eicosanoids is a major contributor to the lethality of the infection. Furthermore, 3D-MSI using high-throughput MALDI TOF was used to generate a 3D model of the host-pathogen lipid interface via the distribution of infection- and host-specific lipids [30]. Similar breakdown products from AA-CLs have also been implicated in nonalcoholic fatty liver disease. In a MALDI MSI study by Hall et al. distinct zonation of PC and SM lipids in nonalcoholic fatty liver tissues was observed. This zonation was subsequently lost following progression to nonalcoholic steatohepatitis [10]. AA-CL signals, in particular $[\mathrm{PC}(36: 4)+\mathrm{K}]^{+}$and $[\mathrm{PC}(38: 4)+\mathrm{K}]^{+}$, were elevated in zone 3 of the liver and strongly correlated with the location of lysophosphatidylcholine acyltransferase 2, a key enzyme involved in phospholipid remodelling and the creation of AA-CLs, using immunohistochemistry. Moreover, proinflammatory eicosanoids were again observed and associated with release of free AA from phospholipids following the action of cytosolic phospholipase A2 and arachidonate 15-lipoxygenase enzymes [10]. The importance of AA-CL's has also resulted in their spatial alterations being observed in several other MSI studies, including a MALDI MSI study detailing elevated levels of [PI(18:0_20:4)-H] $]^{-}$at the outer edges of colorectal cancers [31]. In another study, a logarithmic decrease in AA-CLs signals was observed from the base to the top of colon crypts and again correlated with local expression of various enzymes involved in lipid synthesis, breakdown, and metabolism [32]. Other examples of altered AA-CL distributions include an aggregation of AA-containing PC lipids near the site of spared nerve injuries, which correlated with microglia activation [33], and an observed gradient of AA-containing PCs in the axons of mice neurons, with enriched levels observed within the axon and a proximal-to-distal abundance gradient detected [34].

Non-polar lipids, such as triacylglycerides (TAGs), have also been studied with MSI. In one SIMS study, a $\mathrm{Bi}^{3+}$ ion beam was used to probe intestinal absorption of dietary fat in mice, via analysis of their duodenums after different digestion times following force feeding with cholesterol-enriched sunflower oil [35]. Comparisons of peak ratios following different digestion times revealed selective alterations in certain lipid species. For example, peak ratios of C18containing monoacylglyceride (MAG), DAG, and TAG signals increased with digestion time. Spatially, lipid signals corresponding to those species present at high levels in the oil (C18:2 and C18:1) increased mostly in the enterocytes (likely present as cytosolic lipid droplets), while C18:0, C16:1 and C16:0 carboxylate signals decreased in the lamina propria [35]. Isolation of cytosolic lipid 


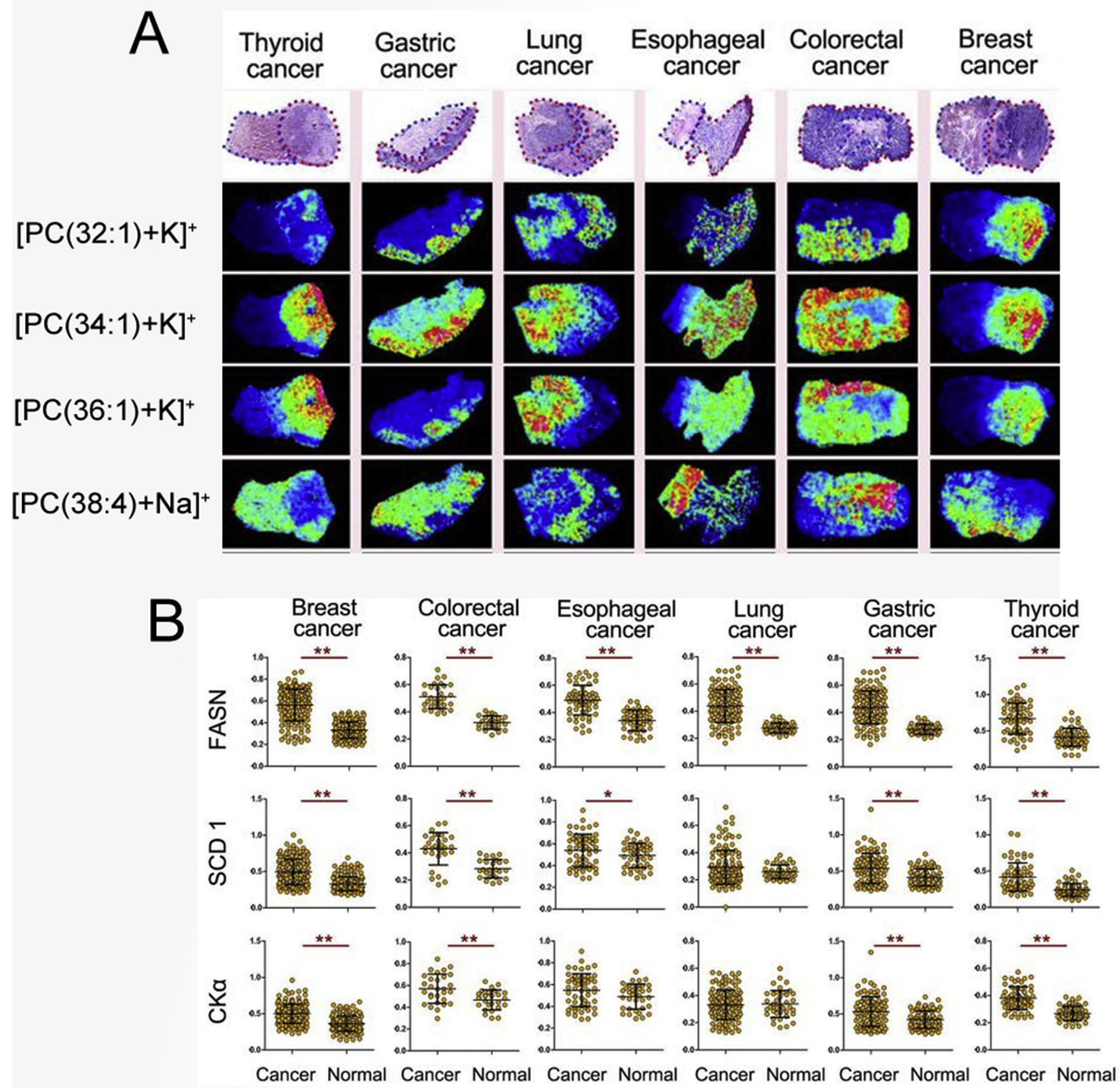

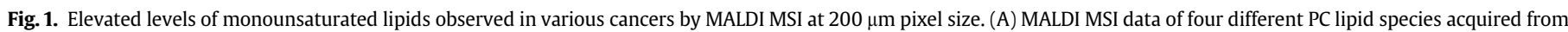

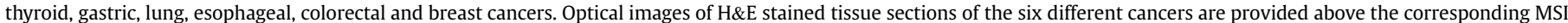

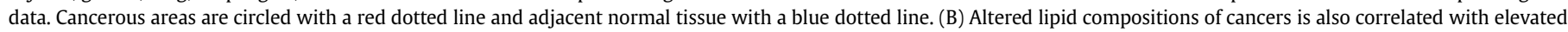

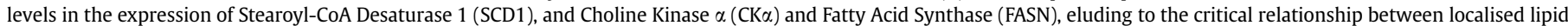
compositions revealed by MSI and enzyme processes (Image adapted with permission from Ref. [25]).

droplets was performed, with the neutral lipid composition of lipid droplets determined with gas-liquid chromatography. Activity of acyl-transferase enzymes within the lipid droplets was also detected (MAG acyl transferase 2 and DAG acyl transferase 2), in addition to other enzymes including acyl-coenzyme A, cholesterol acyltransferase and adipose triglyceride lipase. This suggests that the
SIMS MSI data, which showed localised uptake of dietary lipids, may be rationalised via enzymatic-driven metabolic processes [35]. MSI has also been used to study lipid metabolism in plant seeds. Using MALDI MSI, Horn et al. described the acyl-chain dependencies of lipid distribution in the oil seeds of Camelina sativa [36]. As an example, 18:2 containing lipids, such as PC(16:0_18:2) 
and $\mathrm{PC}(18: 2 / 18: 2)$, were enriched within the embryonic axis relative to cotyledons. In contrast, PC lipids containing $18: 3$ or $20: 1$ chains were enriched in the cotyledons relative to the embryonic axis. A variety of genetically engineered oil seeds, which were enriched in 16:0, 18:1 and 18:2 fatty acids, were produced via interference in the enzymatic synthesis of FAs and subsequently studied with MALDI MSI. In 16:0-enriched seeds, PC(16:0_18:2) revealed a relatively homogenous distribution, in stark contrast to the wild-type seeds [36]. Moreover, even with 16:0 making up 40\% of the available fatty acid pool, little of the fully saturated PC and TAGs (i.e., PC(32:0) or TAG(48:0) were observed, suggesting the presence of a metabolic bottleneck in the generation of fully saturated PC and TAGs. For genetically engineered seeds enriched with 70\% 18:1, PC(18:1/18:1) showed a heterogeneous distribution, being elevated in the cotyledons while polyunsaturated PC lipids were relatively increased in the embryonic axis. These results suggested an incomplete suppression of fatty acid desaturase 2, with it being more active in the embryonic axis. In the context of genetically engineering oil compositions, this could infer an upper limit of 18:1 accumulation determined by compartmentalization. Such effects can only be visualised with imaging methodologies, with this study in particular demonstrating the analytical potential of using altered lipid compositions to infer localised enzymatic processes.

Finally, in some cases one can take advantages of the fact that positive-ion mode lipid MSI often results in the simultaneous observation of multiple adducts for a given lipid species (e.g., $[\mathrm{M}+\mathrm{H}]^{+},[\mathrm{M}+\mathrm{Na}]^{+}$and $\left.[\mathrm{M}+\mathrm{K}]^{+}\right)$. As a result heterogeneity in the local chemical matrix (both endogenous (e.g, salt) and that introduced upon application of the MALDI matrix) can significantly alter the mass spectra acquired from a given position, even for tissue areas with identical lipid compositions. It is thus imperative that changes in adduct ratios are not mistakenly interpreted as altered lipid compositions. However, changes in sodiated and potassiated ion ratios can potentially provide insight into altered activity of $\mathrm{Na}^{+} / \mathrm{K}^{+}$-ATPase activity. For example, using MALDI MSI, a relative increase in $[\mathrm{M}+\mathrm{Na}]^{+}$ion signal of $\mathrm{PC}\left(16: 0 \_18: 1\right)$ compared to $[\mathrm{M}+\mathrm{K}]^{+}$ion has been observed at the site of traumatic brain injury [37], while altered $\mathrm{PC} \mathrm{Na} / \mathrm{K}^{+}$ratios have also been observed in livers following hepatitis B virus (HBV) infected livers with HBVmediated regeneration defects [38].

\section{Adding temporal data: Visualizing local lipid dynamics in tissues}

Despite the vast potential of MSI to study alterations in localised lipid compositions throughout tissues, it is still blind to a critical aspect of lipid biochemistry; that of the dynamic nature of in-vivo lipid synthesis and turnover. A powerful approach to access this dynamic information lies in stable isotope labelling, as employed in flux-based lipidomics studies [39]. By incorporating stable isotopes involved in lipid synthesis into a biological environment, their uptake into various lipids can be detected with MSI. Although the use of stable isotope labelling combined with MSI has found success in SIMS, in particular nano-SIMS which enables sub-cellular imaging of isotopic enrichments, these studies are limited by the extensive fragmentation induced by the desorption/ionisation step (e.g., detection of $\mathrm{CH}^{-}$or $\mathrm{CN}^{-}$) meaning only global enrichments can be measured. It is only recently that soft-ionisation MSI methods been deployed to study lipid dynamics of intact (and thus identifiable) lipid species.

Two studies have applied MSI to study the kinetics of phospholipid synthesis following $\mathrm{D}_{2} \mathrm{O}$ administration $[23,40]$. In both studies, drinking water was enriched with $8 \% \mathrm{D}_{2} \mathrm{O}$, resulting in the incorporation of deuterium into newly synthesised lipids. In the study by Louie et al., mice containing breast tumors were provided access to $8 \% \mathrm{D}_{2} \mathrm{O}$ drinking water for 5 days prior to sacrifice and collection of breast tumor tissue. Analysis of tissue was performed using nanostructured initiator mass spectrometry, which can be viewed as an alternative to MALDI that does not require the sample be coated in an organic matrix, but where the nanostructured surface itself acts as the matrix. The resulting per-pixel TOF spectra provided both lipid compositions (mostly PC lipids) and information regarding local synthesis via the shifted isotope profiles [40]. Distributions of a variety of unlabelled PC lipids are provided in Fig. 3 (row 1 and 4) revealing heterogeneous endogenous lipids throughout the tumor tissue. Interestingly, MSI of the corresponding deuterium enriched lipid signals (Fig. 3 row 2 and 5 ) and fractional enrichment values representing the ratios of labelled:unlabelled lipid species (Fig. 3 row 3 and 6) reveal different ion distributions suggesting area of highest lipid concentrations are not necessarily the areas of highest synthesis rates. These data likely reflect tissue and cell-type specific variations in lipid flux. In a similar study performed with DESI and rat brain tissue, subtle regio-specific alterations in the synthesis of several lipids, including PS(40:6) and PI(38:4) were observed [23]. For example, faster synthesis of PS(40:6) was detected in the cerebral cortex and the septal nucleus. In contrast, $\mathrm{PI}(38: 4)$ revealed the highest turnover in the corpus callosum, where the overall concentration is lowest, providing evidence for either rapid degradation/conversion or redistribution throughout the brain. A potential disadvantage of $\mathrm{D}_{2} \mathrm{O}$ labelling is the high probability that a given lipid species can possess a distribution in the number of deuterium atoms. In the absence of sufficient mass resolution, this can potentially result in isobaric overlaps and masking of signals related to low-abundance lipid species. In an alternative approach, $d_{3}$-palmitate was used to study the kinetics of PC lipid synthesis within neurons cultured in a compartmentalised system [41]. Using high spatial resolution MALDI MSI ( $6 \mu \mathrm{m}$ pixel size), the incorporation of labelled palmitate could be visualised across individual neurons. The most abundant labelled lipid signals corresponded to $\mathrm{PC}(32: 0)$ containing two $\mathrm{d}_{3}$-palmitate acyl chains. Applying $\mathrm{d}_{3}$-palmiate to the loading side of the culture, labelled $\mathrm{d}_{6}-\mathrm{PC}(32: 0)$ was observed around the cell body and also within the neurites along the microgroove regions of the culture. When $d_{3}$-palmiate was only added to the distal side, $d_{6}-P C(32: 0)$ was only enriched in the tips of the neurites [41], suggesting that transport of newly synthesised lipids from the distal side throughout the neuron is restricted.

The above reports have only just begun to scratch the surface of the possibilities enabled by kinetic-MSI coupled with isotope labelling, and it is certain to become a rapidly growing area for MSI in the near future, providing a window into a thus far hidden dimension of biochemical information. It is likely that to fully exploit isotope labelling high mass resolving power MSI will be required to resolve (insofar as is possible with current high resolving power MSI instruments) isobaric ions that will inevitably be present due to both the diversity of naturally generated lipids and those enriched with stable isotopes (i.e., to resolve [PC(34:1)+ $\mathrm{H}^{+}$containing $2 \times$ deuterium or carbon-13 atoms from monoisotopic and $\left.[\mathrm{PC}(34: 0)+\mathrm{H}]^{+}\right)$. Regarding enrichment sensitivity, with current MSI technology it is reasonable to expect the ability to detect and image enrichment factors of $\sim 1 \%$ for many species in a single pixel, which is roughly an order of magnitude higher than required with current extract-based methods (e.g., those based on lipid extraction from homogenised tissue) [42]. With further improvements in sensitivity and dynamic range, isotope labelling strategies will provide a powerful toolbox for studying a diverse array of cell- and tissue-specific lipid synthesis and conversions processes. 
A

Hours Post-Infection (F. novicida)

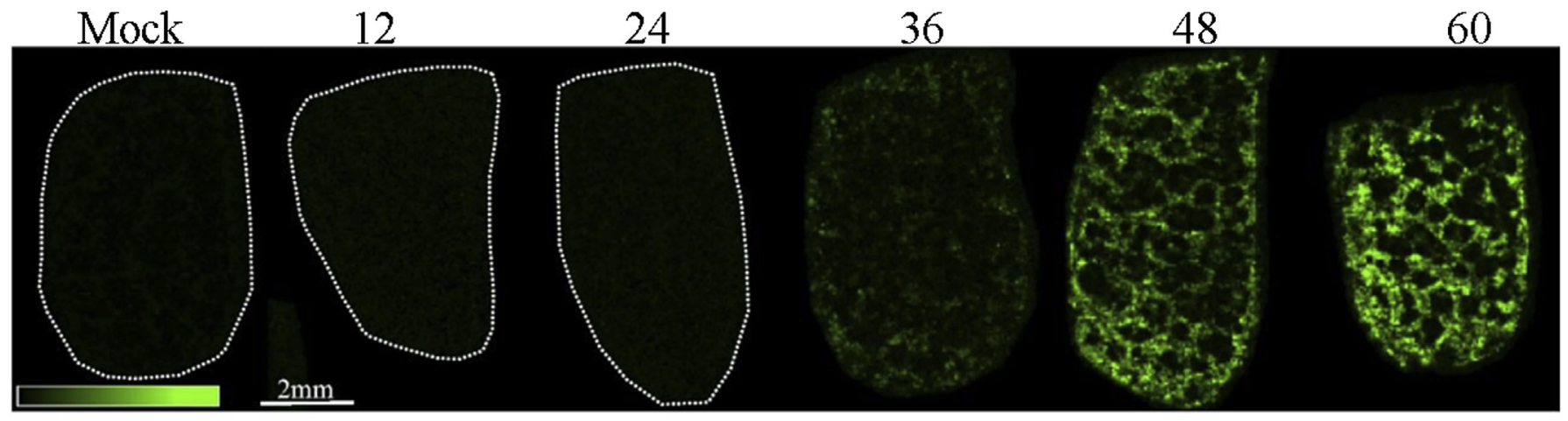

B

Hours Post-Infection (F. novicida)

Mock

12

24

36

48

60

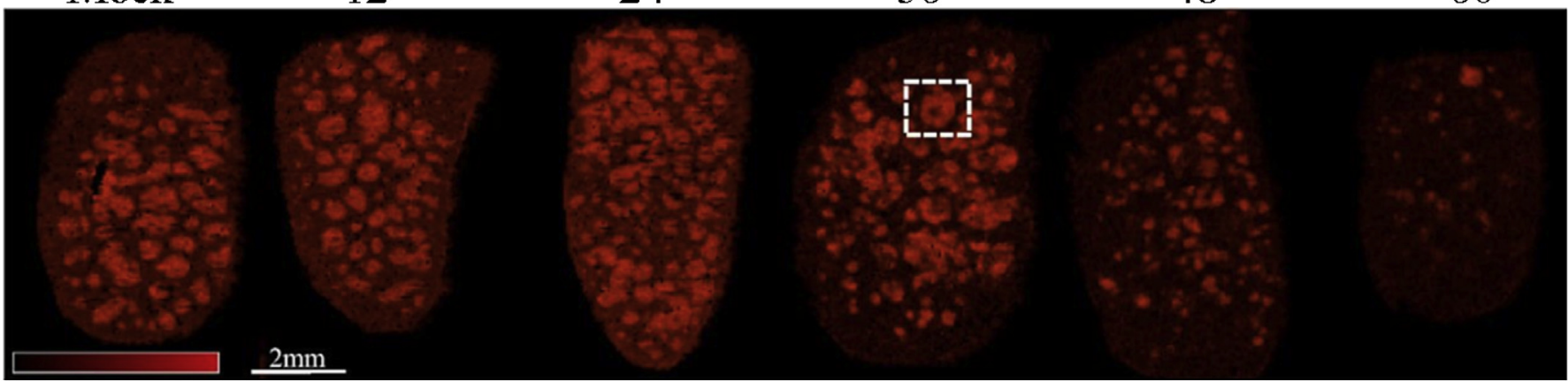

$2 \mathrm{~mm}$

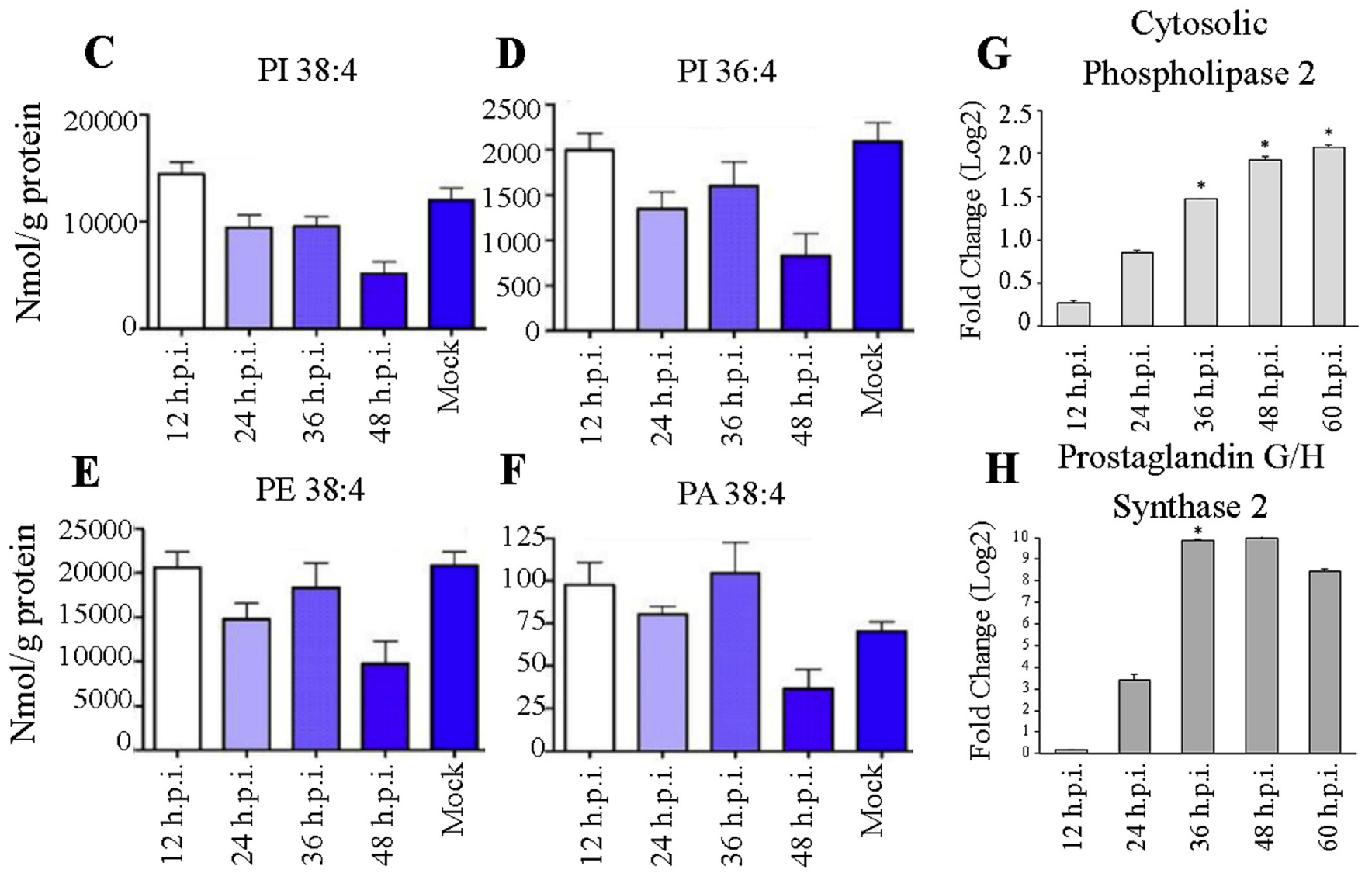



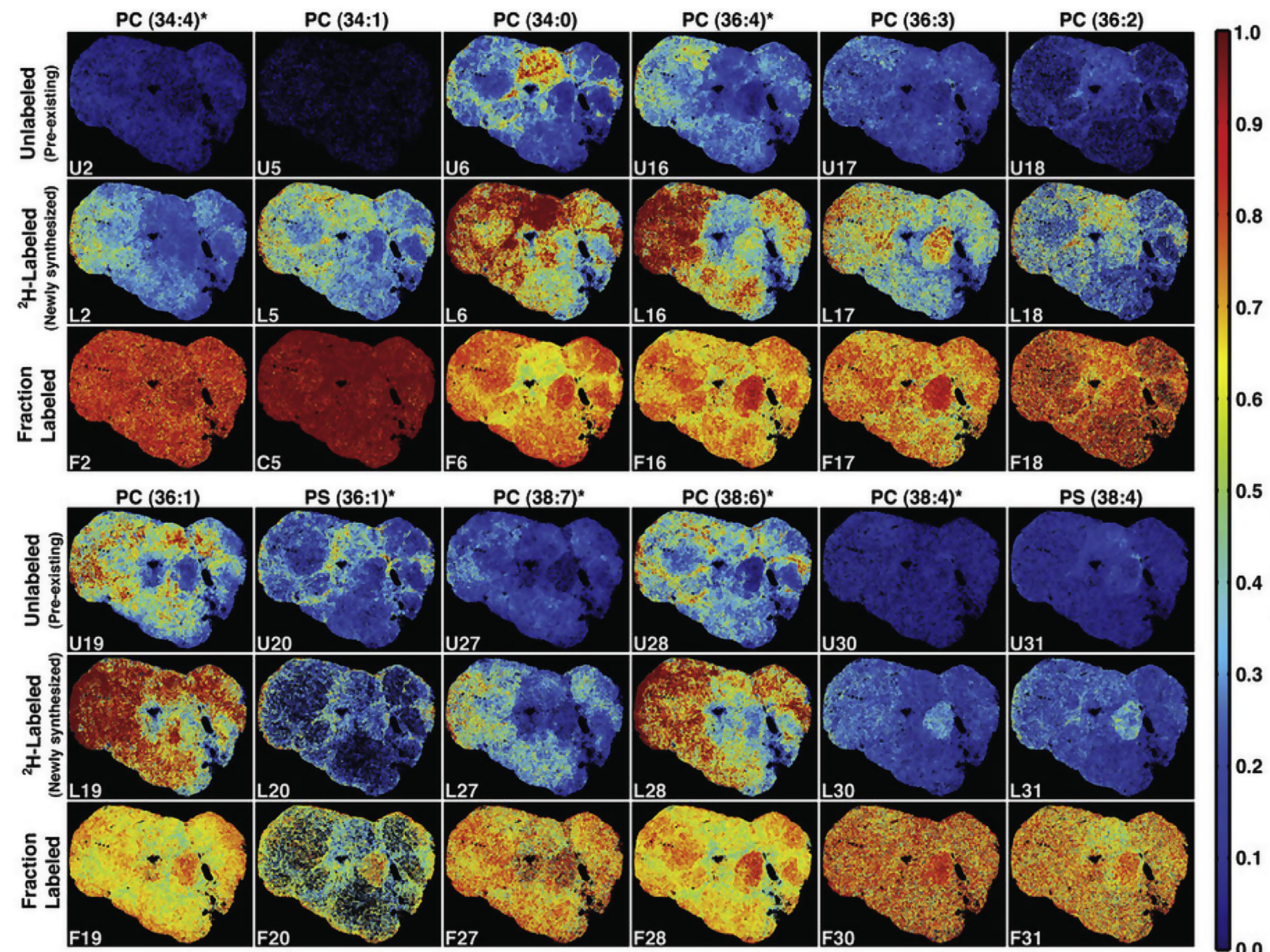

F28

F30

F31

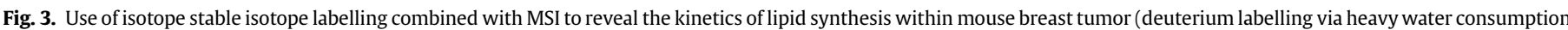

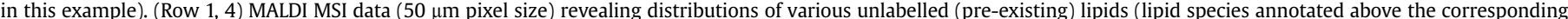

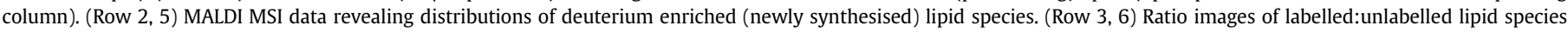
showing relative synthesis rates of various lipids in each pixel (higher intensity equates to faster synthesis and/or slower turnover). Image used with permission from Ref. [40].

\section{Current analytical challenges and future opportunities in lipid MSI}

Lipid MSI has seen a dramatic improvement in both analytical capabilities and biological applications in the last two decades, yet there are still significant hurdles that must be addressed to enable both broad imaging of lipid classes and of well-defined lipid molecules. By large, these issues arise from the vast number of different lipid molecules present in biology. To illustrate, while some lipid classes (e.g., PC and SM in positive ion mode) possess relatively high ionisation efficiencies, other important lipid classes (such as sterols and prostaglandins) are notoriously difficult to ionise with current MSI approaches. Moreover, the complexity of the lipidome introduces significant suppression effects such that the presence of certain lipid classes can impair the detection of others [43].
Lipidome complexity is partly addressed with the emergence of high mass resolution/accuracy MSI using FTICR or Orbitrap analysers. Such approaches facilitate sum-compositional assignment of detected lipid species, but provide little structural information beyond this (e.g., composition of individual fatty acyl chains). Laserinduced post-ionisation (MALDI-2) is as promising approach to address the typically low ionisation efficiencies of some lipid classes $[44,45]$. Combined with a high resolution mass analyser (Orbitrap), MALDI-2 has demonstrated improvements in lipidome coverage for MALDI MSI, with up two-orders of magnitude improvement in signal intensity for a variety of lipid classes possible. For example the dramatic improvement for protonated GalCer and PE lipids in positive-ion mode is shown in Fig. 4, while in negative ion mode large increases in deprotonated signals of a variety of acidic lipid species (e.g., PE, PS, PI, PA and GalCer) are observed $[44,45]$. Other approaches to improve lipid coverage have

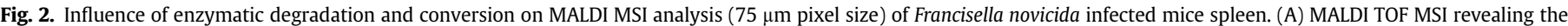

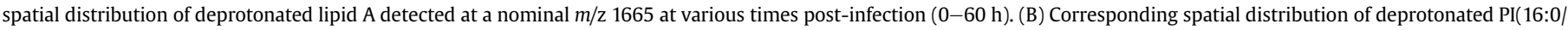

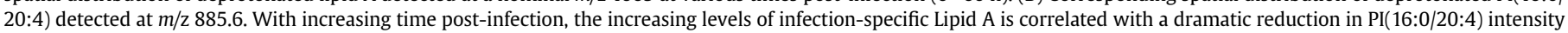

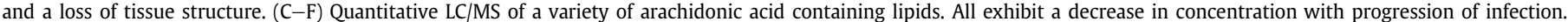

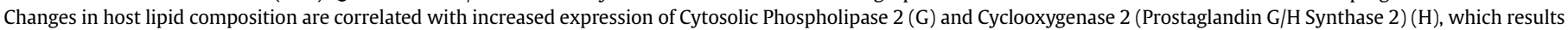
in the release of arachidonic acid from host lipids and their conversion into proinflammatory prostaglandin E2, respectively. Image adapted with permission from Ref. [30]). 


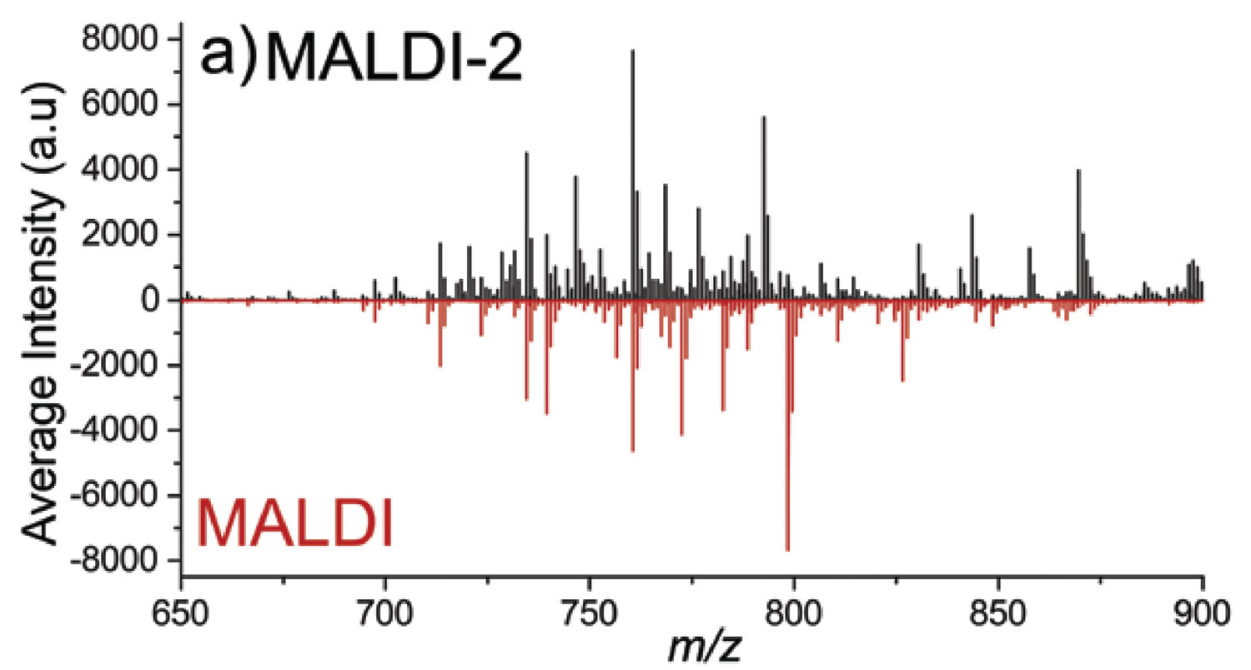

b) $m / z 792.55635$

$\left[\mathrm{PE}\left(18: 0 \_22: 6\right)+\mathrm{H}\right]^{+}$
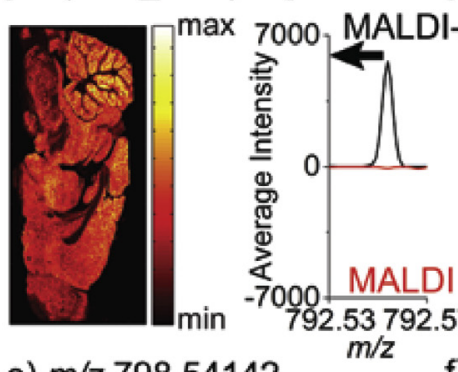

e) $m / z 798.54142$
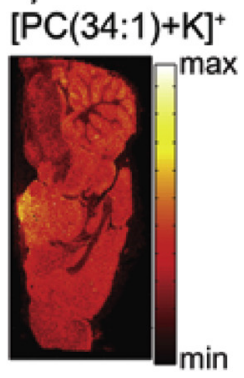

c) $m / z 746.57015$

$$
\begin{aligned}
& \text { [DMPE } \left.\left(16: 0 \_18: 1\right)+\right]^{+}+/ \\
& {\left[\mathrm{PE}\left(18: 0 \_18: 1\right)+\mathrm{H}\right]^{+}}
\end{aligned}
$$

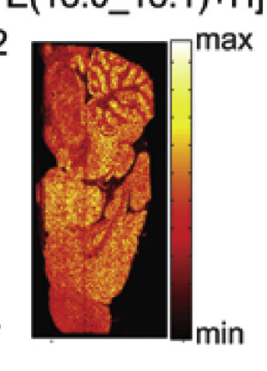

f) $\mathrm{m} / \mathrm{z} 810.68231$

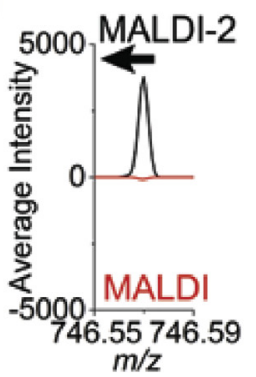

[GalCer(d18:1/24:1)+

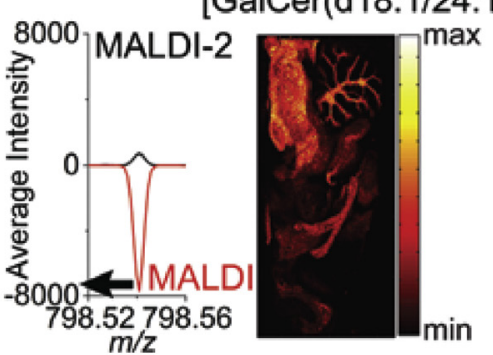

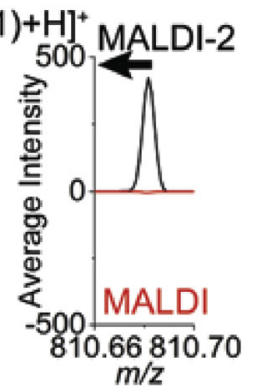

d) $m / z 369.35146$

$\left[\mathrm{Chol}+\mathrm{H}-\mathrm{H}_{2} \mathrm{O}\right]^{+}$

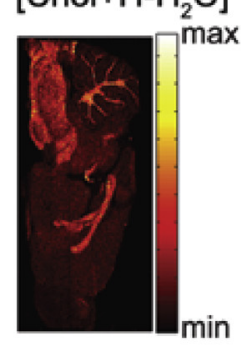

g) H\&E Image
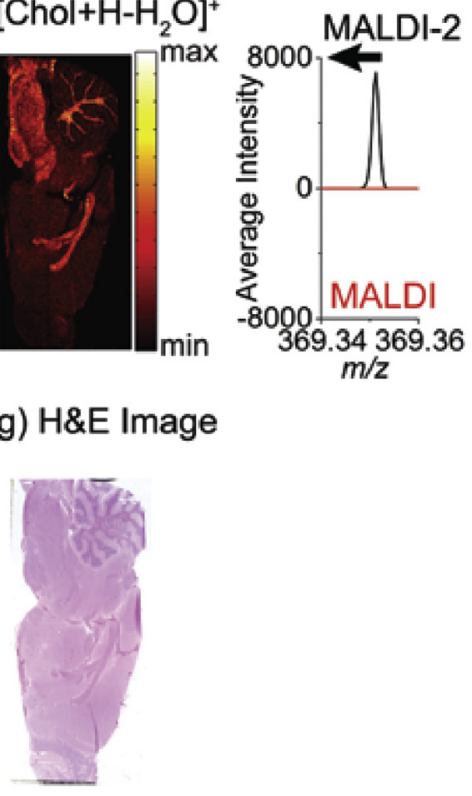

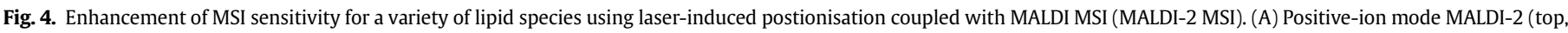

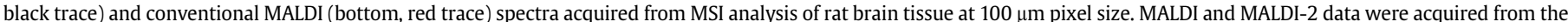

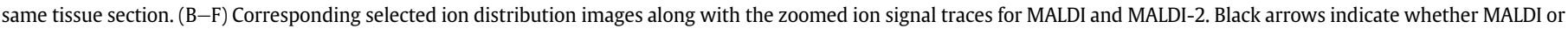

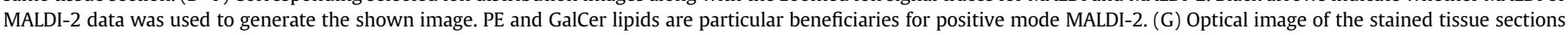
acquired after MSI. Image reproduced with permission from Ref. [45].

been built on the observation that positive and negative-ion mode MSI offers detection of complimentary lipid classes with minimal overlap. For instance, using a precision controlled scanning laser beam and MALDI, it is possible to first acquire positive-ion mode data from a tissue section, with the laser not interacting with the entire tissue surface, after which negative-ion mode data can be acquired from the same tissue section using an interlaced scanning pattern [46]. When applied to zebra-finch and lung cancer xenografts, it was possible to acquire both positive-ion and negative-ion mode data from a $50 \times 50 \mu \mathrm{m}^{2}$ area of tissue and merge the data from both polarities together for multivariate analyses, thus providing more comprehensive lipid coverage from a single sample analysed with MSI. In an alternative approach, Kaya et al. have demonstrated a novel method describing positive and negative-ion lipid MSI, as well as positive mode MSI of $A \beta$ peptides in a transgenic mouse model of Alzheimer's disease (tgSwe) [47]. This approach enabled the spatial correlation of multiple lipid classes and $A \beta$ peptides in amyloid- $\beta$ plaques. Moreover, derivatisation strategies offer an exciting means to study the spatial distributions of lipid not yet detectable with current MSI approaches [48]. While not yet widely applied to lipid MSI, one report by Wu et al. demonstrated using in-situ betaine aldehyde derivatization during DESI-MSI of mouse brain for the enhanced detection cholesterol and Vitamin A [24].

Finally, to fully elucidate the ultimate origin of MSI-detected lipids, knowledge of the precise structure of the lipids is required. Known enzymatic processes give rise to fatty acids with defined chain lengths and double bond positions which are later incorporated into complex lipids [49]. While conventional MS/MS enables identification of acyl chain length and degree of unsaturation, it 
typically provides little information on double bond positions or positioning of fatty acyls on the glycerol backbone. Consequently, the exact identities of lipids in an MSI experiment are ambiguous. In recent years, a selection of alternative strategies such as ozoneinduced dissociation (OzID) [50], ultraviolet photodissociation [51,52], photochemical Paterno-Buchi reactions [53], and ion mobility [54] have been developed to resolve lipid isomers from solution-phase extracts. At present only OzID has been combined with MSI to study the spatial distributions of isomeric lipids [55]. In this study the distributions of both double bond-positional and snpositional isomers were studied in both healthy rat brain and a medulloblastoma mouse model. These data demonstrated that in many instances isomeric lipids have distinct spatial distributions throughout tissues that conventional MSI approaches are blind to. Importantly such techniques enabling imaging of defined isomeric species are likely essential to facilitate correlations of lipid distributions with certain enzymatic processes involved in the selective synthesis and catabolism of individual lipid isomers. This includes the roles of desaturases in synthesis of double bond-positional isomers [49] and phospholipase A1 and A2 enzymes involved in both phospholipid breakdown and the synthesis of polyunsaturated-containing phospholipids.

\section{Conclusions}

MSI has evolved in the last two decades from a molecular imaging technique in its infancy to a highly promising method for studying the spatial distributions of lipids in a diverse array of sample types. However, as discussed here it is only recently that insights into the underlying biochemical origin of lipid spatial distributions have been reported. In-line with general knowledge of lipid biochemistry it is apparent that to understand the biological context of lipids, lipidomics alone is often not enough. In particular, localised enzymatic processes have been heavily implicated as a key determinant of both lipid composition and spatial distributions within tissues. Such multimodal information is likely a necessity to understand the cause and effect of lipid composition within tissues. However, lipids should not only be viewed as the end-products of enzymatic processes. Lipid composition is also determined by the local availability of molecular building blocks, which can be influenced via a variety of factors (i.e., diet or medications) and can be directly correlated to disease pathology. Additionally, lipids themselves can participate in a multitude of metabolic/signalling processes and strongly influence membrane properties.

With ever increasing analytical performance, the ability to acquire new spatially-resolved lipidomics information has also emerged. This includes isotope labelling studies enabling localised lipid dynamics to be probed, as well as advances in desorption/ ionisation methods permitting enhanced sensitivity and broader detection of lipid classes during MSI experiments. Such increased lipid coverage is essential to understand the spatial correlations amongst different, structurally related lipid species. In the near future, we envisage further adoption and adaptation of methods originally developed for extract-based lipidomics which provided enhanced structural detail and resolution. This is a key step towards overcoming the current ambiguity in MSI lipid annotations and will facilitate the imaging of structurally-defined lipid molecules. Such advances will be essential to understand the effect of desired membrane properties, that are sensitive to subtle structural alterations [56] and on the signalling networks leading to synthesis of certain lipids. Like any method however, lipid MSI is not without its future challenges. Two of the most significant are the development of quantitative MSI workflows that enable absolute surface concentrations of lipids to be determined, and the somewhat related effects of ion suppression that can potentially mean relative ion distributions across different tissue types may not reflect absolute or relative local concentrations. Nonetheless both the development of quantitative MSI approaches and understanding the relationships between absolute concentrations and ion distributions with MSI are active areas of research [57,58].

Finally, we hope to reiterate that despite the challenges still be addressed, lipids present a highly promising and detectable family of molecules for MSI studies, both for tissue classification/diagnostics (i.e., based on lipid profile) and for understanding the underlying biochemical processes occurring throughout biology.

\section{Acknowledgements}

This work has been made possible with the financial support of the Dutch province of Limburg through the LINK program. The authors also acknowledge funding from Interreg V EMR and the Netherlands Ministry of Economic Affairs within the "EURLIPIDS" project (project number EMR23).

\section{References}

[1] M.R. Wenk, The emerging field of lipidomics, Nat. Rev. Drug Discov. 4 (2005) 594-610.

[2] S.J. Blanksby, T.W. Mitchell, Advances in mass spectrometry for lipidomics, Annu. Rev. Anal. Chem. (Palo Alto Calif) 3 (2010) 433-465.

[3] A. Shevchenko, K. Simons, Lipidomics: coming to grips with lipid diversity, Nat. Rev. Mol. Cell Biol. 11 (2010) 593-598.

[4] L.A. McDonnell, R.M. Heeren, Imaging mass spectrometry, Mass Spectrom. Rev. 26 (2007) 606-643.

[5] K.A. Berry, J.A. Hankin, R.M. Barkley, J.M. Spraggins, R.M. Caprioli, R.C. Murphy, MALDI imaging of lipid biochemistry in tissues by mass spectrometry, Chem. Rev. 111 (2011) 6491-6512.

[6] M.K. Passarelli, N. Winograd, Lipid imaging with time-of-flight secondary ion mass spectrometry (ToF-SIMS), Biochim. Biophys. Acta 1811 (2011) 976-990.

[7] L.S. Eberlin, C.R. Ferreira, A.L. Dill, D.R. Ifa, R.G. Cooks, Desorption electrospray ionization mass spectrometry for lipid characterization and biological tissue imaging, Biochim. Biophys. Acta Mol. Cell Biol. Lipids 1811 (2011) 946-960.

[8] M. Sugimoto, M. Wakabayashi, Y. Shimizu, T. Yoshioka, K. Higashino, Y. Numata, T. Okuda, S. Zhao, S. Sakai, Y. Igarashi, Y. Kuge, Imaging mass spectrometry reveals acyl-chain- and region-specific sphingolipid metabolism in the kidneys of sphingomyelin synthase 2-deficient mice, PLoS One 11 (2016), e0152191.

[9] L.S. Eberlin, I. Norton, A.L. Dill, A.J. Golby, K.L. Ligon, S. Santagata, R.G. Cooks, N.Y. Agar, Classifying human brain tumors by lipid imaging with mass spectrometry, Cancer Res. 72 (2012) 645-654.

[10] Z. Hall, N.J. Bond, T. Ashmore, F. Sanders, Z. Ament, X. Wang, A.J. Murray, E. Bellafante, S. Virtue, A. Vidal-Puig, M. Allison, S.E. Davies, A. Koulman, M. Vacca, J.L. Griffin, Lipid zonation and phospholipid remodeling in nonalcoholic fatty liver disease, Hepatology 65 (2017) 1165-1180.

[11] J. Balog, L. Sasi-Szabo, J. Kinross, M.R. Lewis, L.J. Muirhead, K. Veselkov, R. Mirnezami, B. Dezso, L. Damjanovich, A. Darzi, J.K. Nicholson, Z. Takats, Intraoperative tissue identification using rapid evaporative ionization mass spectrometry, Sci. Transl. Med. 5 (2013), 194ra193.

[12] L.S. Eberlin, I. Norton, D. Orringer, I.F. Dunn, X. Liu, J.L. Ide, A.K. Jarmusch, K.L. Ligon, F.A. Jolesz, A.J. Golby, S. Santagata, N.Y. Agar, R.G. Cooks, Ambient mass spectrometry for the intraoperative molecular diagnosis of human brain tumors, Proc. Natl. Acad. Sci. USA 110 (2013) 1611-1616.

[13] J.L. Norris, R.M. Caprioli, Analysis of tissue specimens by matrix-assisted laser desorption/ionization imaging mass spectrometry in biological and clinical research, Chem. Rev. 113 (2013) 2309-2342.

[14] R.J. Goodwin, Sample preparation for mass spectrometry imaging: small mistakes can lead to big consequences, J. Proteom. 75 (2012) 4893-4911.

[15] M. Kompauer, S. Heiles, B. Spengler, Atmospheric pressure MALDI mass spectrometry imaging of tissues and cells at $1.4-\mu \mathrm{m}$ lateral resolution, Nat. Methods 14 (2017) 90-96.

[16] S.G. Ostrowski, C.T. Van Bell, N. Winograd, A.G. Ewing, Mass spectrometric imaging of highly curved membranes during Tetrahymena mating, Science 305 (2004) 71-73.

[17] H. Tian, D.A. Six, T. Krucker, J.A. Leeds, N. Winograd, Subcellular chemical imaging of antibiotics in single bacteria using C60-secondary ion mass spectrometry, Anal. Chem. 89 (2017) 5050-5057.

[18] T.B. Angerer, Y. Magnusson, G. Landberg, J.S. Fletcher, Lipid heterogeneity resulting from fatty acid processing in the human breast cancer microenvironment identified by GCIB-ToF-SIMS imaging, Anal. Chem. 88 (2016) $11946-11954$.

[19] J.S. Fletcher, S. Rabbani, A. Henderson, P. Blenkinsopp, S.P. Thompson, N.P. Lockyer, J.C. Vickerman, A new dynamic in mass spectral imaging of single biological cells, Anal. Chem. 80 (2008) 9058-9064. 
[20] G.L. Fisher, A.L. Bruinen, N. Ogrinc Potocnik, J.S. Hammond, S.R. Bryan, P.E. Larson, R.M. Heeren, A new method and mass spectrometer design for TOF-SIMS parallel imaging MS/MS, Anal. Chem. 88 (2016) 6433-6440.

[21] M.K. Passarelli, A. Pirkl, R. Moellers, D. Grinfeld, F. Kollmer, R. Havelund, C.F. Newman, P.S. Marshall, H. Arlinghaus, M.R. Alexander, A. West, S. Horning, E. Niehuis, A. Makarov, C.T. Dollery, I.S. Gilmore, The 3D OrbiSIMS-label-free metabolic imaging with subcellular lateral resolution and high mass-resolving power, Nat. Methods 14 (2017) 1175.

[22] C. Wu, A.L. Dill, L.S. Eberlin, R.G. Cooks, D.R. Ifa, Mass spectrometry imaging under ambient conditions, Mass Spectrom. Rev. 32 (2013) 218-243.

[23] R.H. Carson, C.R. Lewis, M.N. Erickson, A.P. Zagieboylo, B.C. Naylor, K.W. Li, P.B. Farnsworth, J.C. Price, Imaging regiospecific lipid turnover in mouse brain with desorption electrospray ionization mass spectrometry, J. Lipid Res. 58 (2017) 1884-1892.

[24] C. Wu, D.R. Ifa, N.E. Manicke, R.G. Cooks, Rapid, direct analysis of cholesterol by charge labeling in reactive desorption electrospray ionization, Anal. Chem. 81 (2009) 7618-7624.

[25] S. Guo, Y. Wang, D. Zhou, Z. Li, Significantly increased monounsaturated lipids relative to polyunsaturated lipids in six types of cancer microenvironment are observed by mass spectrometry imaging. Sci. Rep. 4 (2014) 5959.

[26] C.M. Paton, J.M. Ntambi, Biochemical and physiological function of stearoylCoA desaturase, Am. J. Physiol. Endocrinol. Metab. 297 (2009) E28-E37.

[27] Y. Ide, M. Waki, T. Hayasaka, T. Nishio, Y. Morita, H. Tanaka, T. Sasaki, K. Koizumi, R. Matsunuma, Y. Hosokawa, H. Ogura, N. Shiiya, M. Setou, Human breast cancer tissues contain abundant phosphatidylcholine(36ratio1) with high stearoyl-CoA desaturase-1 expression, PLoS One 8 (2013), e61204.

[28] L.S. Eberlin, M. Gabay, A.C. Fan, A.M. Gouw, R.J. Tibshirani, D.W. Felsher, R.N. Zare, Alteration of the lipid profile in lymphomas induced by MYC overexpression, Proc. Natl. Acad. Sci. USA 111 (2014) 10450-10455.

[29] Y. Liu, Y. Chen, A. Momin, R. Shaner, E. Wang, N.J. Bowen, L.V. Matyunina, L.D. Walker, J.F. McDonald, M.C. Sullards, A.H. Merrill Jr., Elevation of sulfatides in ovarian cancer: an integrated transcriptomic and lipidomic analysis including tissue-imaging mass spectrometry, Mol. Cancer 9 (2010) 186.

[30] A.J. Scott, J.M. Post, R. Lerner, S.R. Ellis, J. Lieberman, K.A. Shirey, R.M.A. Heeren, L. Bindila, R.K. Ernst, Host-based lipid inflammation drives pathogenesis in Francisella infection, Proc. Natl. Acad. Sci. USA 114 (2017) 12596-12601.

[31] T. Hiraide, K. Ikegami, T. Sakaguchi, Y. Morita, T. Hayasaka, N. Masaki, M. Waki, E. Sugiyama, S. Shinriki, M. Takeda, Y. Shibasaki, S. Miyazaki, H. Kikuchi, H. Okuyama, M. Inoue, M. Setou, H. Konno, Accumulation of arachidonic acidcontaining phosphatidylinositol at the outer edge of colorectal cancer, Sci. Rep. 6 (2016) 29935.

[32] J. Bestard-Escalas, J. Garate, A. Maimo-Barcelo, R. Fernandez, D.H. Lopez, S. Lage, R. Reigada, S. Khorrami, D. Ginard, J. Reyes, I. Amengual, J.A. Fernandez, G. Barcelo-Coblijn, Lipid fingerprint image accurately conveys human colon cell pathophysiologic state: a solid candidate as biomarker, Biochim. Biophys. Acta 1861 (2016) 1942-1950.

[33] T. Banno, T. Omura, N. Masaki, H. Arima, D. Xu, A. Okamoto, M. Costigan, A. Latremoliere, Y. Matsuyama, M. Setou, Arachidonic acid containing phosphatidylcholine increases due to microglial activation in ipsilateral spinal dorsal horn following spared sciatic nerve injury, PLoS One 12 (2017), e0177595.

[34] H.J. Yang, Y. Sugiura, K. Ikegami, Y. Konishi, M. Setou, Axonal gradient of arachidonic acid-containing phosphatidylcholine and its dependence on actin dynamics, J. Biol. Chem. 287 (2012) 5290-5300.

[35] A. Seyer, M. Cantiello, J. Bertrand-Michel, V. Roques, M. Nauze, V. Bezirard, X. Collet, D. Touboul, A. Brunelle, C. Comera, Lipidomic and spatio-temporal imaging of fat by mass spectrometry in mice duodenum during lipid digestion, PLoS One 8 (2013), e58224.

[36] P.J. Horn, J.E. Silva, D. Anderson, J. Fuchs, L. Borisjuk, T.J. Nazarenus, V. Shulaev, E.B. Cahoon, K.D. Chapman, Imaging heterogeneity of membrane and storage lipids in transgenic Camelina sativa seeds with altered fatty acid profiles, Plant J. 76 (2013) 138-150.

[37] J. Hankin, S. Farias, R. Barkley, K. Heidenreich, L. Frey, K. Hamazaki, H.-Y. Kim, R. Murphy, MALDI mass spectrometric imaging of lipids in rat brain injury models, J. Am. Soc. Mass. Spectrom. 22 (2011) 1014-1021.

[38] E.S. Park, J.H. Lee, J.H. Hong, Y.K. Park, J.W. Lee, W.J. Lee, J.W. Lee, K.P. Kim, K.H. Kim, Phosphatidylcholine alteration identified using MALDI imaging MS in HBV-infected mouse livers and virus-mediated regeneration defects, PLoS One 9 (2014), e103955.

[39] A.D. Postle, A.N. Hunt, Dynamic lipidomics with stable isotope labelling, J. Chromatogr. B Anal. Technol. Biomed. Life Sci. 877 (2009) 2716-2721.

[40] K.B. Louie, B.P. Bowen, S. McAlhany, Y. Huang, J.C. Price, J.H. Mao, M. Hellerstein, T.R. Northen, Mass spectrometry imaging for in situ kinetic histochemistry, Sci. Rep. 3 (2013) 1656.

[41] E. Sugiyama, I. Yao, M. Setou, Visualization of local phosphatidylcholine synthesis within hippocampal neurons using a compartmentalized culture system and imaging mass spectrometry, Biochem. Biophys. Res. Commun. 495 (2018) 1048-1054.

[42] J. Brandsma, A.P. Bailey, G. Koster, A.P. Gould, A.D. Postle, Stable isotope analysis of dynamic lipidomics, Biochim. Biophys. Acta 1862 (2017) 792-796.

[43] M. Petkovic, J. Schiller, M. Müller, S. Benard, S. Reichl, K. Arnold, J. Arnhold, Detection of individual phospholipids in lipid mixtures by matrix-assisted laser desorption/ionization time-of-flight mass spectrometry: phosphatidylcholine prevents the detection of further species, Anal. Biochem. 289 (2001) 202-216.

[44] J. Soltwisch, H. Kettling, S. Vens-Cappell, M. Wiegelmann, J. Muthing, K. Dreisewerd, Mass spectrometry imaging with laser-induced postionization, Science 348 (2015) 211-215.

[45] S.R. Ellis, J. Soltwisch, M.R.L. Paine, K. Dreisewerd, R.M.A. Heeren, Laser postionisation combined with a high resolving power orbitrap mass spectrometer for enhanced MALDI-MS imaging of lipids, Chem. Commun. 53 (2017) 7246-7249.

[46] S.R. Ellis, J. Cappell, N.O. Potocnik, B. Balluff, J. Hamaide, A. Van der Linden, R.M. Heeren, More from less: high-throughput dual polarity lipid imaging of biological tissues, The Analyst 141 (2016) 3832-3841.

[47] I. Kaya, D. Brinet, W. Michno, M. Baskurt, H. Zetterberg, K. Blenow, J. Hanrieder, Novel trimodal MALDI imaging mass spectrometry (IMS3) at 10 mum reveals spatial lipid and peptide correlates implicated in abeta plaque pathology in Alzheimer's disease, ACS Chem. Neurosci. 8 (2017) 2778-2790.

[48] E. Ryan, G.E. Reid, Chemical derivatization and ultrahigh resolution and accurate mass spectrometry strategies for "shotgun" lipidome analysis, Acc. Chem. Res. 49 (2016) 1596-1604.

[49] H. Guillou, D. Zadravec, P.G. Martin, A. Jacobsson, The key roles of elongases and desaturases in mammalian fatty acid metabolism: insights from transgenic mice, Prog. Lipid Res. 49 (2010) 186-199.

[50] H.T. Pham, A.T. Maccarone, M.C. Thomas, J.L. Campbell, T.W. Mitchell, S.J. Blanksby, Structural characterization of glycerophospholipids by combinations of ozone- and collision-induced dissociation mass spectrometry: the next step towards "top-down" lipidomics, The Analyst 139 (2014) 204-214.

[51] P.E. Williams, D.R. Klein, S.M. Greer, J.S. Brodbelt, Pinpointing double bond and snpositions in glycerophospholipids via hybrid $193 \mathrm{~nm}$ ultraviolet photodissociation (UVPD) mass spectrometry, J. Am. Chem. Soc. 139 (2017) 15681-15690.

[52] E. Ryan, C.Q.N. Nguyen, C. Shiea, G.E. Reid, Detailed structural characterization of sphingolipids via $193 \mathrm{~nm}$ ultraviolet photodissociation and ultra high resolution tandem mass spectrometry, J. Am. Soc. Mass Spectrom. 28 (2017) 1406-1419.

[53] X. Ma, Y. Xia, Pinpointing double bonds in lipids by Paternò-Büchi reactions and mass spectrometry, Angew. Chem. Int. Ed. 53 (2014) 2592-2596.

[54] M. Groessl, S. Graf, R. Knochenmuss, High resolution ion mobility-mass spectrometry for separation and identification of isomeric lipids, The Analyst 140 (2015) 6904-6911.

[55] M.R.L. Paine, B.L.J. Poad, G.B. Eijkel, D.L. Marshall, S.J. Blanksby, R.M.A. Heeren, S.R. Ellis, Mass spectrometry imaging with isomeric resolution enabled by ozone-induced dissociation, Angew. Chem. Int. Ed. 57 (2018) 10530-10534.

[56] H. Martinez-Seara, T. Róg, M. Pasenkiewicz-Gierula, I. Vattulainen, M. Karttunen, R. Reigada, Interplay of unsaturated phospholipids and cholesterol in membranes: effect of the double-bond position, Biophys. J. 95 (2008) 3295-3305.

[57] S.R. Ellis, A.L. Bruinen, R.M.A. Heeren, A critical evaluation of the current stateof-the-art in quantitative imaging mass spectrometry, Anal. Bioanal. Chem. 406 (2014) 1275-1289.

[58] J.A. Hankin, R.C. Murphy, Relationship between MALDI IMS intensity and measured quantity of selected phospholipids in rat brain sections, Anal. Chem. 82 (2010) 8476-8484. 